\title{
Research on the Development of China's Urban Development Based on the Ecological Concept of Low-carbon
}

\author{
Dong Junyan ${ }^{1,2, a}$, Li Kechao ${ }^{1, b}$, Xing Yuenan ${ }^{1}$,Tan Tenglong ${ }^{1}$ \\ ${ }^{1}$ School of Architecture and Design, Changchun Institute of Technology, Changchun, China \\ ${ }^{2}$ School of Architecture, Harbin Institute of Technology, Harbin, China \\ adjyrob@gmail.com, ’93045611@qq.com
}

\section{Keywords: Low-carbon, Urban, Environment, Ecological concept}

Abstract. The rapid progress of urbanization in China will be an important event that will affect the economic and social development of China and the world. The development of low-carbon eco city should study the low carbon eco city development strategy, determine the development of low-carbon eco city, follow the city function and low-carbon eco city development, rational development of city industry; scientific planning, reasonable guide the development of low-carbon eco city planning, improve the index system, the implementation of the planning EIA to ensure sustainable development of the city; at the same time sustainable transportation system comprehensive guidance city energy efficient operation, the construction of sustainable city traffic system; research on energy saving technology promotion, the promotion of green building technology and clean production technology, to provide technical support for the development of low-carbon eco city; promote the system innovation, the reform of the taxation system and evaluation system, and create a system environment for the development of low-carbon eco city, reform the administrative management system of city government, city government function transformation and optimization, to promote the development of low-carbon eco city.

\section{Introduction}

In most cities, the daily life of urban dwellers is separated from natural ecosystem. Most of the people living in cities will think that water comes from the tap, milk comes from the bottle, food comes from the supermarket, waste dropped into dustbin." [1] Because of our lack of emotional awareness to the nature and the area between the edge of the city, I easily slip into the thinking framework of separating the urban and rural areas, considered them as two distinct entity, which has separate geographical unit".

The expansion during the industrial age, both comes from and strengthens the two basic habit of thinking: fragmentation and objectification. When we considered the world composed of discrete objects, the habit of fragmentation comes into being_—we see a chair, forgetting the forest, trees, rain and the carpenter making a chair. It led to the habit of fragmentation, when we concern ourselves as separated from the outside world, and forgetting the interdependence of life. [2] It forms the habit of objectification, when we forget the existence of other life around us. It is impossible to change this reality, if the way of we think, which led to these imbalances, couldn't be change. "We must learn how the natural ecosystem operating again, so that we can transform our society into a simulation of natural ecosystems."

The relationship between human society and nature has also experienced the process of balance imbalance - rebalancing. There are serious imbalance between the city as artificial environment and natural ecological environment after the rapid development of industrialization, it shows that the natural environment was one-dimensional looted and destructed by the city, and its capacity can't maintain the original pattern of urban development. Therefore, in the post-industrial society, we need to find a new pattern of urban development, which maintains the balance between human society and natural environment. 


\section{Key ecological urban agglomeration}

American scholar Lorenz had made his famous "butterfly effect" theory that a butterfly incite wings in Brazil will cause a tornado in Texas. This seemingly easy joke very subtle reflects the whole Earth's ecosystem relevance. Any regions, cities and individuals can't exist independently from the environment, it coincides with the holistic thinking of "harmony of man with nature" in our traditional culture, and the Taoist emphasizing on the "all things orderly", "Inaction and balance" concept.

Urban and natural environment has always been a whole, and they correlated overall. There are no city can operate alone without natural environment. The correlation of Urban and natural environment stresses associating with overall urban functions, structure, time, space and geographical factors. This requires people to understand the variables as much as possible and establish an integrated planning and design ideas. We should take the principle of "whole before others", emphasizing the coordination of People, artificial elements and natural elements, integrating natural and artificial environments through material, energy, information and other ways, and considering it from the function (physical, ecological, economic, social, energy, etc.), spatial level (regional level, city level, area level, lot level, and the different climatic regions), the concept of time (long-term,medium-term and short-term), management decision (at all levels departments).It means that all the relevant content, including social, economic, technological, environmental and management factors was integrated, all levels and scales environmental design was related from the perspective of system integration, respecting nature, emphasizing the whole instead section. [3]

The global expansion of the cities has entered a new earth "niche" after Industrial revolution. New York, London, Paris, Shanghai, Tokyo as a global great metropolis, with its advantages of Capital, technology and scale, has a superior "niche" in the global city network, dominating the worldwide resources. However, after the city obtained these resources from the nature, what can cities feedback to the natural environment, and such as these access and feedback can sustainable? Looking back at history, we can find that there were many human civilizations flourished for many centuries or even millennia, such as our Chinese civilization. However, this continuity of human civilization is the results that it found the way to harmony with other living systems. When man and nature coexist in harmony, our needs of food, water, energy and waste disposal could continue be meet, and our philosophy and religion have guided us, let us feel and understand our own position in a larger network of lives.

Among city itself, city and city, city and country, city and regional, there exists a relationship of complex integrated interdependence in natural, social and economic aspects. From the view of ecological, In addition to interaction of urban ecological communities and physical environment, cities have capacity of energy flow, material cycling and information transmission, while being restricted by social production, relations of production, and the superstructure associated with it. Low-carbon city is not a closed system, but a social, economic and natural complex in a certain area, only the city with a complex ecosystem worked well is the low-carbon city. Between the complex ecosystems within the city and the larger complex regional ecosystems, it requires cities constituted integrity, diversity, openness and symbiosis required by a typical "ecosystem", being able to coordinate, grow and develop, flourish commonly with good self-organizing capacity, so the city can be called a truly low-carbon city.

Low-carbon city is not only a simple natural ecology, but a human ecology, that is, characterized by education, technology, culture, ethics, law and systems into a comprehensive low-carbon, respecting low-carbon values, low carbon philosophy, low-carbon ethics and low-carbon conscious awareness, forming a resource-conserving system of social production and consumption. We should establish a justice, equality, safe and comfortable social environment, which has conscious protection of the environment and the mechanism of promoting self-development of human. The harmony between man and nature is the core concepts of social value system in the future. It will go beyond anthropocentrism to promote reflection and self-examination of human, re-assessing the history and thinking about the future. 


\section{Strategies for developing low carbon cities}

There is no absolute model of development, and the diversification of geographical, economic and political will always make balance and rich the different path of human development. However, due to the needs of sustainable human development, we must look for a harmony development model between cities and natural environment, which could meet the diverse needs of the population, but also maintain our environment from destruction, and ultimately realizing the ideal of low-carbon city_ urban development with nature, not against nature.

A new model of urban development emerges, being bound to take another pattern of urban development away from the stage of history as the background, from agricultural city to industrial city that, from the industrial city to low-carbon city is also the case, associated with this a technology innovation. Low-carbon city is a development model, and low-carbon technologies are powerful tools, technological innovation will bring new opportunities to the development of cities and its natural environment. Our major impact on the natural environment depends on the type of urban development and the maintenance of the technology used in the development of cities, reducing carbon emissions is the core of low-carbon urban development, and "reduce, recycle, absorption, generation" is a effective method.

Traditional urban planning reduced the complex city to many "functional area" to analyze the economic geographical location with the typical approach of rational machine, while low-carbon urban planning concerned a variety of materials, energy input and output in this system, looking the city and the natural environment as a whole. Although low-carbon city has an open structure, but it can't infinitely extend in the city scale. An urban structure of low-carbon-based is highly dynamic, open system, which is easy to innovate without affecting its balance.

Contrast with the lack of considering traffic and the disorder of regional development pattern of the city, a more rational development pattern of low-carbon city should be corridor model combined by the guide rail or regional public transport, so as to achieve energy conservation goals through spatial integration and control the number of cars. Map of the city is expanding and gradually compact at the same time. The spread of the city is no longer unlimited, and space form shifted from the scattered layout to the corridor-like layout (Fig1). The main working and living areas of people distribute along the city's public transport network. The changes of low-carbon life concept and low-carbon technologies will gradually change people's lifestyles and living space. "Must review the basis of the current urban form, and it is very important to re-discover the inherent qualities and characteristics of the places our daily lives with the ecological perspective." We need to in-depth analysis of the change process and the various factors, so that we can understand the new law of the formation of spatial structure and its rationality.
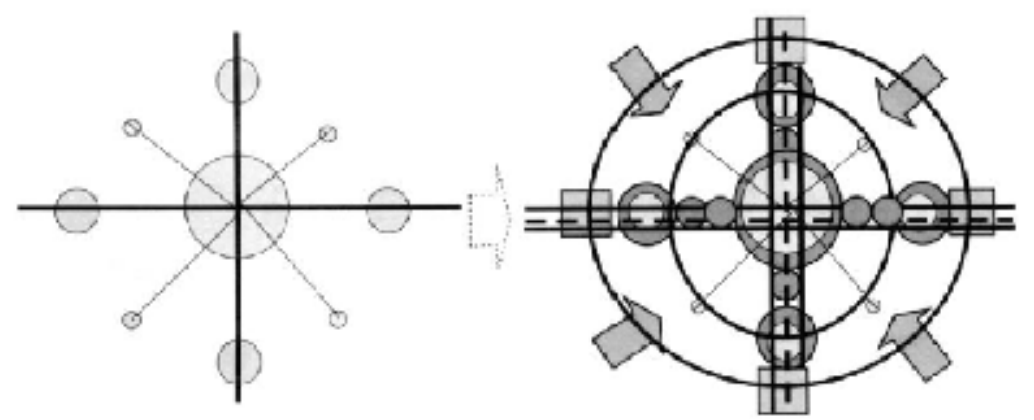

Fig.1 Modes of public transport corridors and low-carbon urban form

The low-carbon development of city is a complex social change, which also involves low-carbon city as development model and low-carbon technologies as a powerful tool, it is the complex pattern formed by social, economic and environmental objectives constitutes low-carbon city development goals, changing the social, urban and regional.

The features of a low carbon society show: the globalization of energy saving behavior, low-carbon lives become a daily way of life. Due to the promotion of low-carbon technologies, the 
old economic system and urban structure are rearranged. The overall construction of urban low-carbon technology needs synchronization integration of social and spatial planning, in the cultural, economic and political three levels:

Culturally, the city is defined by the region, and their personality should be protected, their historical roots should be maintained. In the low-carbon era, the traditional social mechanisms of the city will gradually be transformed, people need to adapt the new work and life organization, while ensuring that city maintain adequate communication between the eco-units, overcoming the tendency to tribalism.

Economically, the city and the region must find their new role in low-carbon economy. In the low-carbon economy, the main source of productivity is energy saving and emission reduction, so from the local economy, the industrial structure to the workforce skills, all these needed to find a new role in low-carbon urban space.

Politically, in the process of build low-carbon cities, local governments play a central role. Only by strengthening its role, could local government exert influence to the economic and political organizations. if the city government organizations to encourage the implementation of low carbon economy and social development policies, they will be able to grasp the tremendous power brought by low-carbon urban development. The point of the policy may be expressed as a utopia; but sometimes, however, the view of utopia will shake institutional perspective short-sighted, and enhance their consciousness of social transformation and social control.

\section{Conclusions}

Low-carbon city development should keep on towards the vibrant, healthy, creative goal. We need to think the relationship of man and nature, city and earth deeply, couldn't neglect the impact to the future by choosing the model of urban development activities and daily life style. We are faced with the choice of urban development mode, which has far-reaching effects on the future of Earth and the health of all life on Earth. So when we keep respecting things that we can't build, and respecting all the things that Mother Earth gives us, we will not only construct ourselves a home, but also develop a future for all future generations.

\section{Acknowledgements}

This work was financially supported by Youth Foundation of Changchun Institute of Technology (No.320140006), JiLin Provincial Social Science Foundation (No. 2015BS83) and Teaching reform project of Changchun Institute of Technology.

\section{References}

[1] Junyan Dong, Hong Jin. The design strategy of green rural housing of Tibetan areas in Yunnan, China. Renewable Energy, Vol. 49, pp. 63-67.

[2] Junyan Dong, Wen Cheng. Based on the Characteristics of Respondents and the Voice of the Urban Neighborhood Public Space Business Facilities Noise Environment Evaluation Research. Journal of Harbin Institute of Technology.2014,20(4),pp.103-109.

[3] Junyan Dong, Hong Jin, Jian Kang, Xi Chen, A pilot study of the acoustic environment in residential areas in Harbin, towards the questionnaire design. Journal of Harbin Institute of Technology.2011,18(2),pp.319-322. 